\title{
Hacia una reconstrucción de los conflictos de la memoria. El caso del Museo de la Memoria y los Derechos Humanos en Chile
}

\author{
Towards a Reconstruction of the Conflicts of Memory. The Case of the Museum of Memory and \\ Human Rights in Chile
}

\author{
Mauro Basaure \\ Escuela de Sociología, Universidad Andrés Bello, Chile \\ Centro de Estudios de Conflicto y Cohesión Social, COES, Chile
}

\section{RESUMEN}

El artículo sostiene que la memoria juega un rol intermediario entre el presente político y la historia. Puesto que el presente político es intrínsecamente un escenario de disputa, la memoria transfiere esa disputa a la historia, al atribuir políticamente significación cognitiva o normativa a eventos o períodos seleccionados. Propongo que de esto emergen cuatro formas de la memoria distintas y potencialmente controversiales, estas son, una forma cognitiva positiva y otra negativa, por un lado; una forma sociointegrativa positiva y otra negativa, por otro. La relación conflictiva entre ellas define lo que llamo controversias o disputas de la memoria. Por medio de una revisión de distintas reflexiones teóricas sobre la relación entre política, historia y memoria, construyo primero un modelo original para el análisis de las disputas de la memoria y luego lo aplico a la controversia pública respecto de la instalación y funcionamiento del Museo de la Memoria y los Derechos Humanos de Chile entre 2007 y 2016. El artículo concluye que la crítica de posiciones políticas concernientes a la relación entre política e historia produce distintas formas de la memoria con énfasis cognitivo o socio-integrativo que colisionan entre sí produciendo una trayectoria de la memoria cambiante en el tiempo.

Palabras Clave: Memoria; Derechos Humanos; Controversia; Crítica; Museo de la Memoria

\section{ABSTRACT}

The article asserts that memory plays an intermediary role between political present and history. As political present is intrinsically a matter of dispute, memory transfers this dispute into history by politically attributing cognitive or normative significance to selected events and periods. I argue that four different and potentially controversial forms of memory emerge from this, namely, a positive and a negative cognitive form of memory, on the one hand, and a negative and a positive socio-integrative form, on the other hand. The conflictive link between these forms of memory defines what I call controversies or disputes of memory. By elaborating on different theoretical reflections about the relation between politics, history, and memory, I firstly construe an original framework to analyze disputes of memory and subsequently I apply this to the public controversy between 2007 and 2016 regarding the installation and functioning of the Chilean Museum of Memory and Human Rights. The article concludes that criticisms of political positions concerning the relation between politics and history produce different and colliding forms of cognitive or socio-integrative memory, which creates a fluctuating time trajectory for memory.

KEYWORDS: Memory; Human Rights; Controversy; Critique; Museum of Memory

MAD, N³7 (2017), PP. 113-142

DOI: 10.5354/0718-0527.2017.47278

(C) CC BY-NC 3.0 CL 


\section{INTRODUCCIÓN ${ }^{1}$}

Las modernas teorías de la memoria (social, política, cultural, colectiva) coinciden en sostener que, en la actualidad, la memoria no puede ser entendida como un regreso al pasado, como una fuente contenedora de hechos, informaciones o datos con pretensión de objetividad, los cuales reflejarían el modo en que acontecieron las cosas. Por el contrario, la misma idea de memoria presupone un componente selectivo y, por tanto, activo que se despliega en el presente como forma de interpretación y reconstrucción del pasado, y que sirve a distintos grupos sociales como esquema de inclusión y orientación de la acción (Assmann 2006; Habermas 1992; Halbwachs 1997; Hobsbawm \& Ranger 1983; Koselleck 2003; Luhmann 1996, 1997; Nassehi 2008).

Dos consecuencias interrelacionadas surgen de lo anterior. La primera es que, si la memoria no es un contenedor de hechos independiente de las selecciones presentes, entonces la memoria es una actualización que depende de los actores que le dan forma, cuestión que coincide con las tesis básicas de la sociología accionalista e interpretativa. De esto resulta que existan distintas construcciones selectivas de memoria sobre eventos del pasado que permiten orientar la acción de grupos sociales de modos alternativos (Luhmann 1996). La segunda consecuencia surge de conectar las construcciones alternativas de la memoria con el presente político. Si la memoria contribuye a la orientación de la acción en el presente, entonces ella desarrolla una suerte de mediación entre pasado histórico y presente político (Habermas 1992; Halbwachs 1997). De este modo, la orientación de la acción que la memoria logra derivar de una lectura política de la historia que, en tanto política, abre un terreno de controversia sobre la apropiación del pasado es lo que en este artículo denomino 'disputas de la memoria'.

Una primera hipótesis de este artículo es, entonces, que las disputas de la memoria pueden ser entendidas y estudiadas como controversias entre posiciones basadas en distintas 'formas sociales abstractas' de construir la relación entre historia y política por medio de la memoria. Rescato el con-

\footnotetext{
${ }^{1}$ El autor agradece al Fondo Nacional de Ciencia y Tecnología, Fondecyt, Proyecto 1140344 y en especial la colaboración del Co-investigador de dicho proyecto, el Dr. Aldo Mascareño El autor agradece también al proyecto Fondecyt 1150790, del cual es co-investigador y al Centro de Estudios de Conflicto y Cohesión Social, COES, Conicyt/Fondap/15130009. También a gradece a Hans Joas por su invitación a realizar una estadía de investigación en la Universidad Humboldt de Berlín en el marco de la beca Ernst-Troeltsch-Honorarprofessur, en el transcurso el autor trabajó en los detalles últimos de este artículo.
} 
cepto de "forma" de la sociología de Georg Simmel (2009), en tanto relación social general con contenidos diversos. Al considerar las disputas de la memoria como formas sociales indico que estas constituyen un efecto de relaciones recíprocas entre individuos que acontecen históricamente y cuyo contenido puede ser diverso de acuerdo con distintas circunstancias temporales (vinculación con momentos) y espaciales (vinculación con lugares). Es decir, los contenidos de la memoria dependen de situaciones sociales históricas a las que hay que agregar un contenido emocional, en tanto la memoria evoca "interacciones sociales fuertemente emotivas" (Simmel 2009: 561). En segundo lugar, aun cuando la memoria tenga ese sustrato particular vinculado a situaciones sociales (momentos más lugares), ella siempre se construye en relaciones sociales que pueden ser capturadas en formas sociales por medio de las cuales los individuos pueden situar su experiencia en un contexto mayor que otorga sentido a la interacción y a sus contenidos específicos. En tanto forma social, la memoria puede por tanto orientar en el presente la acción social de acuerdo a la valoración del contenido histórico de esa experiencia. En tercer lugar, la valoración del contenido histórico de la experiencia se realiza igualmente en la interacción y -según la hipótesisen la esfera de la interacción política, de manera tal que las formas sociales abstractas de la memoria constituyen una mediación entre situaciones sociales de contenido histórico y el presente político en el cual tiene lugar la interacción social y sus consecuencias recíprocas entre individuos.

En otros términos, sostengo que las disputas de la memoria son disputas por el posicionamiento político de la historia en el horizonte del presente. Siendo así, se hace necesario determinar cuáles son las formas de la memoria de manera tal que ellas produzcan, en el presente, una disputa de la memoria. Este artículo argumenta que la reconstrucción de la memoria, en tanto relación entre historia y política en el presente, puede seguir una forma cognitiva o una socio-integrativa $y$, en ambos casos, la orientación de la acción puede ser positiva (acción afirmativa) o negativa.

Una segunda hipótesis de este artículo, derivada de la anterior, se asocia con el estudio de caso: la controversia en torno al Museo de la Memoria y los Derechos Humanos (en adelante, MMDH) entre los años 2007 (año de propuesta de creación del Museo) y 2016. Sostengo que esta controversia es, en último término, una controversia entre distintas formas de la memoria en tres fases históricas: a) en una primera fase, entre la forma socio-integrativa negativa que es el fundamento de la acción del Museo (apropiación 
crítica de la historia) y una neutralización de la memoria; b) en una segunda fase, entre la forma socio-integrativa negativa y la forma cognitiva negativa (advertencia contextualizada hacia futuro); y c) en una tercera fase, entre la forma socio-integrativa negativa y una forma socio-integrativa positiva (construcción de una cultura general de derechos humanos y valores democráticos).

Para desplegar estas proposiciones, el artículo propone en la primera sección un modelo conceptual original de disputas entre formas de la memoria, distinguiendo entre su dimensión cognitiva (positiva y negativa) y su dimensión socio-integrativa (positiva y negativa) en base a distintas perspectivas de la literatura sobre el tema que sustentan la propuesta analítica. Luego de ello, en la segunda sección, expongo brevemente algunas consideraciones metodológicas para entonces poner el modelo a prueba en su capacidad interpretativa por medio del análisis de la controversia suscitada a propósito de la creación e instalación del $\mathrm{MMDH}$ en Chile desde el año 2007 en adelante. En la sección final establezco las principales conclusiones del análisis en relación con las hipótesis planteadas.

\section{LAS FORMAS DE LA MEMORIA}

$\mathrm{Si}$, como la he definido en la introducción, la memoria es un dispositivo de mediación selectiva entre pasado histórico y presente político que contribuye a la orientación de la acción en el presente, entonces las formas de la memoria configuran una esfera de relaciones entre historia y política que puede adquirir modalidades distintas. Sostengo que estas formas de la memoria son una 'forma cognitiva' (positiva y negativa) y una 'forma socio-integrativa' (positiva y negativa).

En primer lugar, se puede emplear la historia como 'fuente de conocimiento' para la construcción política del futuro. A esta relación entre historia y política la denomino una forma de mediación de 'orden cognitivo'. Según ella, el acceso al pasado, a la relación entre antecedentes pasados y consecuentes pasados, sería útil como guía para direccionar los destinos de la sociedad, para imitar o evitar lo ya ocurrido teniendo como base la comparación entre el pasado y el presente. Los actores que recurren a esta forma normalmente se autocomprenden como si tuviesen un acceso privilegiado al pasado. Ejemplo clásico es Cicerón (1862): la historia como maestra de la vida (historia magistra vitae), pilar central no sólo de la historiografía clásica, sino que también de la medieval, en la concepción escolástica 
de la historia, del renacimiento en el pensamiento de Maquiavelo (Castaño 2013), e incluso en enfoques contemporáneos comprendidos bajo la fórmula de Habermas: "aprender de las catástrofes" (Habermas 1995, 2001; Koselleck 1992).

No sin ciertas dificultades, es posible diferenciar analíticamente de esta forma cognitiva una segunda forma de relación entre historia y política. En ella, el uso de la historia se entiende como 'fuente de solidaridad e integración social' pues los eventos del pasado son fuente de conmemoración de cara a la construcción de una identidad colectiva. A esta relación entre historia y política la denomino una forma de mediación de 'orden socio-integrativo'. De acuerdo con ella, el acceso al pasado permite un tipo de relación, ya sea de identificación o de desidentificación con él, aunque en ambos casos lo que está en juego es la producción de un tipo específico de integración social y de estabilización de determinadas expectativas normativas que no alcanzan a ser cubiertas al interior de la sistémica calculabilidad jurídica (Mascareño 2012, 2013; Kastner 2012, 2015). La sociología clásica es fuente clave aquí: Émile Durkheim (2003) y sobre todo su discípulo Maurice Halbwachs (1997), con su trabajo sobre la memoria colectiva. Asimismo, Jürgen Habermas (1995), por medio de su idea de uso público de la historia, y Hauke Brunkhorst (2005), por medio del concepto de solidaridad, caben dentro de esta forma socio-integrativa de la memoria.

Puesto que se trata de formas de la memoria y, por tanto, de distintas modalidades de relación entre historia y política, se deben suponer diferencias en cuanto a los 'contenidos' de ellas. Mientras que el primer tipo de relación (cognitiva) ha sido mayormente investigado (Koselleck 1992, 2003), sobre todo en su fuente clásica representada por Thomas Hobbes (Basaure 2001, Basaure 2013b), no ocurre lo mismo con el segundo tipo de relación (socio-integrativa). Antes de abordar esto, y con el fin de dar un tono conceptualmente más acabado a esta diferencia de relacionamiento entre historia y política -y perfilar de mejor modo las formas de las disputas de la memoria-, se requiere introducir una nueva distinción, tanto en la relación cognitiva como en la socio-integrativa. Esta busca rescatar la significación positiva o negativa que la interpretación política de los actores, a través de la memoria, da a la historia.

En tal sentido, si el evento de memoria al que refiere la forma cognitiva es considerado 'positivo', entonces la historia es típicamente vista como un 'ejemplo o modelo de acción a seguir en el presente'. En cambio, si el 
evento de memoria es considerado como 'negativo', su uso político en tanto memoria se expresará típicamente bajo la forma de la 'advertencia para la acción presente de cara a la construcción futura de la sociedad' - es decir, que, ante circunstancias reconociblemente (o potencialmente) similares, no debe cometerse el error pasado.

Del mismo modo cabe analizar la forma socio-integrativa de relación entre historia y política. Si el evento de memoria al que refiere esta forma es considerado 'negativo', su uso político en tanto memoria se expresará típicamente 'como objeto de reflexión autocrítica' respecto de la identidad colectiva. En otros términos, el evento negativo es integrado en la identidad con el fin de generar una autorreflexión que contribuya a la construcción futura de la comunidad. Esta es típicamente la forma del Nunca Más. Si, por el contrario, el evento es considerado como 'positivo', esa forma se expresa típicamente como la conmemoración de eventos y afirmación de valores con los que 'los individuos deben identificarse' de cara a la continuidad de una tradición sobre cuyas bases se produce la identidad social del colectivo. La Tabla 1 sintetiza estos modos distintos de apropiación del pasado de cara a la construcción política y cultural de la sociedad futura

Tabla 1. Formas de relación entre historia y política

\begin{tabular}{|c|c|c|c|c|}
\hline $\begin{array}{c}\text { Forma de la } \\
\text { memoria }\end{array}$ & \multicolumn{2}{|c|}{ Cognitiva } & \multicolumn{2}{c|}{ Socio-integrativa } \\
\hline Valoración & Positiva & Negativa & Negativa & Positiva \\
\hline Contribución & $\begin{array}{c}\text { Ejemplaridad, } \\
\text { modelo exitoso } \\
\text { de acción }\end{array}$ & $\begin{array}{c}\text { Advertencia de } \\
\text { cara a la acción } \\
\text { futura }\end{array}$ & $\begin{array}{c}\text { Autorreflexión } \\
\text { crítica, apropia- } \\
\text { ción crítica de la } \\
\text { historia }\end{array}$ & $\begin{array}{c}\text { Identificación } \\
\text { con la tradición y } \\
\text { afirmación de } \\
\text { valores }\end{array}$ \\
\hline $\begin{array}{c}\text { Predominio } \\
\text { histórico }\end{array}$ & $\begin{array}{c}\text { Pierde rele- } \\
\text { vancia pública } \\
\text { en siglo XX y } \\
\text { se transforma } \\
\text { en discusión de } \\
\text { expertos }\end{array}$ & $\begin{array}{c}\text { Tiende a } \\
\text { prevalecer } \\
\text { desde } \\
\text { la segunda } \\
\text { mitad del siglo } \\
\text { XX }\end{array}$ & $\begin{array}{c}\text { Tiende a prevale- } \\
\text { cer desde la se- } \\
\text { gunda mitad del } \\
\text { siglo XX }\end{array}$ & $\begin{array}{c}\text { Conmemoración } \\
\text { ritualizada del } \\
\text { siglo XIX y XX; } \\
\text { formación de } \\
\text { tradición de de- } \\
\text { rechos humanos } \\
\text { en siglo XX }\end{array}$ \\
\hline
\end{tabular}

Fuente: Elaboración propia

La descripción de estas formas de la memoria no tiene un carácter puramente lógico. Por el contrario, la distinción positivo/negativo con que se 
pueden manifestar las mencionadas formas de relación entre historia y política pueden ser rastreadas históricamente, siguiendo sus desplazamientos de acento. A continuación, las caracterizo en base a la literatura especializada.

\subsection{FORMA COGNITIVA POSITIVA}

En la forma cognitiva positiva de memoria, la historia, en tanto fuente de conocimiento, es considerada en su sentido de ejemplaridad y modelo exitoso de acción. Se trata de una experiencia vicaria: lo que no puede ser experimentado por un individuo, debe remitir a la experiencia de otros. Es esta experiencia ajena la que queda en disposición de ser apropiada como modelo de la acción propia. En palabras de Reinhart Koselleck (1992: 39), la historia (Historie) nos pone en la posición de "repetir los éxitos del pasado en vez de caer, en el presente, en errores anteriores". Para lograr este efecto, la historia debía concebirse como simple suma de relatos singulares de las que pudiera extraerse la ejemplaridad. La historia es, en este caso, historia de personalidades y acontecimientos ilustrativos.

Según Koselleck (2004), esta forma de comprensión de la historia se sostiene hasta el siglo XVIII, cuando la historia adquiere un mayor grado de abstracción que trasciende los hechos singulares. En tal situación, la historia es concebida como un 'singular colectivo' constituido por capas que se entrelazan en el movimiento histórico. El movimiento histórico se autonomiza de las historias particulares y se consuma en la filosofía de la historia, la que, más bien producto de su capacidad de generalización, puede ser situada en la forma socio-integrativa positiva.

\subsection{FORMA COGNITIVA NEGATIVA}

En relación con la forma cognitiva negativa, tres ejemplos sustentan su presencia. Hobbes (1981), quien escribe su obra de cara a las guerras religiosas intestinas de la Inglaterra de su época, entiende a la historia, a la experiencia del pasado, como "prudencia"; esto es, como una fuente de sabiduría para una acción presente, de cara a la construcción de la paz futura de la sociedad. La experiencia indica qué es lo que conduce a la paz y qué a la guerra (de Jouvenel 1959; Basaure 2001, 2013). En esta misma tradición, Edmund Burke (2004: 355) escribe críticamente sobre los hechos de la revolución francesa, considerándolos como perversos y manifestando el deseo de que 
la historia sea fuente de aprendizaje del futuro: "Tal es el efecto de la perversión de la historia [...] Pero la historia, en el siglo XIX, mejor entendida y empleada, enseñará a la posteridad civilizada -espero- a aborrecer las fechorías de esa era bárbara".

Durante el siglo XX -la era de los extremos y donde se pone en cuestión la idea de progreso lineal de la historia reflejado especialmente en la filosofía de la historia-, se instala con mayor peso esta codificación negativa del vínculo cognitivo entre historia y política. Una de sus expresiones más relevantes puede ser resumida en la pregunta '¿aprender de las catástrofes?', realizada por intelectuales alemanes post Segunda Guerra Mundial como Micha Brumlik (2004) y Jürgen Habermas (1995, 2001), en términos de una invitación a revisar críticamente la historia del corto siglo XX, precisamente con el objeto de no repetirla. Sin duda, esta reflexión remite a un componente socio-integrativo sintetizado en la fórmula del 'aprendizaje normativo' (que reviso más adelante), pero el punto clave aquí para entender una dimensión de esta reflexión como forma cognitiva negativa de la memoria, es justamente la negación del aspecto positivo de la forma historia magistra vitae y su reconversión en una forma de evitación: "La historia puede fungir como maestra (Lebrmeisterin) sólo en tanto instancia crítica. En el mejor de los casos nos dice cómo no debemos hacer las cosas" (Habermas 1995: 187). La historia, negativamente codificada, se constituye como advertencia, como ejemplo negativo, a partir de la revisión de una tradición fracasada, de las posibilidades que la construcción política del presente y del futuro no puede heredar.

En una dimensión distinta, pero bajo la misma forma cognitivo negativa de la memoria, desde fines del siglo XX en adelante la creciente reflexión sobre riesgos en alta tecnología y desastres naturales ha empleado la misma pregunta en tono afirmativo: 'aprender de las catástrofes' (Hoffman 2008; Kunreuther \& Useem 2010). En este caso, no se trata de una tradición política específica fracasada, sino del fracaso de la política como horizonte cognitivo de regulación de desarrollos técnicos (Perrow 1984), desastres naturales (Topper \& Lagadec 2013) y de problemas transnacionales como el terrorismo (Perrow 2007) -problemas que, por su alta complejidad, se transforman en fuente de peligro, no solo para comunidades locales, sino que se expanden como riesgos globales (Beck 2010). El foco se traslada a la política bajo la fórmula de la identificación de vulnerabilidades y a la descentralización de las capacidades de reacción. Las catástrofes enseñan, en principio, la forma de acción que hay que evitar, pues esta ha originado la 
catástrofe. La memoria media entre la catástrofe histórica y las medidas políticas que se deben tomar para no cometer los errores del pasado. Olvidar o ignorar el pasado establece un horizonte de amenaza y asedio permanente al presente.

\subsection{FORMA SOCIO-INTEGRATIVA NEGATIVA}

En la forma socio-integrativa negativa de la memoria, la historia, en tanto fuente de solidaridad e integración social, es considerada como horizonte de reflexión autocrítica en relación con la construcción de la identidad colectiva en el presente. Sin duda, Habermas se inscribe, a este respecto, dentro de una tradición que prosigue una vertiente normativa de la idea de integración y solidaridad social. Ello, sin embargo, en el marco de una transformación de su sentido original, más bien positivo. Implicado directamente en -y en cierto sentido provocador de- la llamada 'disputa de los historiadores' (Historikerstreit) que tuvo lugar en Alemania en los años 80 a propósito del origen del holocausto judío, Habermas entiende a la conmemoración -el traer a memoria de modo oficial- en el marco del complejo proceso de construcción de una conciencia nacional autocrítica, es decir, en un sentido negativo. Tal conciencia es, además, para él, uno de los pasos pragmáticos claves en el proceso de construcción de una ética común de los derechos humanos. Constituyendo estos, junto con la democracia, el contenido básico de las constituciones liberales y la herencia fundamental de la revolución francesa, dice Habermas, dicha nueva conciencia autocrítica es potencialmente una base para una nueva forma de patriotismo; uno basado, ya no en la identificación con los valores particularistas de la nación, sino en la identificación con el mencionado núcleo normativo básico de las constituciones liberales modernas. Se trata de un patriotismo constitucional ( $\mathrm{Ha}-$ bermas 1992; Basaure 2014).

$\mathrm{El}$ derecho adquiere, en este proyecto habermasiano, una fuerza integrativa fundamental: patriotismo constitucional implica la identificación ciudadana con los valores universalistas del sistema democrático y los derechos humanos; identificación a la que el recuerdo permanente de la tragedia histórica de su violación resultaría vital. Ahora bien, más que la tesis habermasiana del patriotismo constitucional, lo relevante aquí es que -pese a la radicalidad de los quiebres y las catástrofes sociales de los Estados nacionales modernos- Habermas rescata, desde un punto de vista de sociología 
normativa, la posibilidad de una solidaridad con base en la identificación nacional, pero exige de ella que se haga con base en la propia memoria reflexiva de esos quiebres y tragedias nacionales y no en una mera conmemoración positiva con eventos orientados a la (re)producción continuista de una identificación con la tradición nacionalista, propia del siglo XIX y la primera mitad del XX. La perspectiva de Habermas expresa a nivel conceptual una tendencia general de desplazamiento 'desde' la conmemoración positiva a lo que se ha llamado el 'deber de memoria' (devoir de mémoire), la obligación oficial y estatalmente instituida de recordar la tragedia en el marco de una 'política del pasado' (Vergangenheitspolitik). Esta tendencia se expresa en un cambio de la cultura de la conmemoración y de los monumentos: desde la monumentalidad heroico-patriótica del siglo XIX a la monumentalidad de la memoria de las tragedias del siglo XX. "Política del pasado" (Ruderer 2010) o "política de arrepentimiento" (Olick 2007), son nombres que invocan este espíritu.

Una modalidad adicional de la forma socio-integrativa negativa es la que recurre públicamente a la semántica cristiana del perdón como apropiación crítica de un pasado traumático. Esta forma se construiría en torno al reconocimiento de la culpa que una comunidad tendría por haber permitido el horror en su historia, e indicaría, mediante símbolos, retóricas y rituales, lo que esa comunidad política (incluso global) no puede repetir en la construcción de su presente y futuro (Meier 2010). El punto ha sido objeto de controversia por dos razones. Primero, la lógica del perdón invocada en nombre de otros está siempre sometida a cálculos políticos o jurídicos (reconciliación, amnistía) que trivializan la intimidad del perdón; segundo, la mundialización de esta forma socio-integrativa negativa violenta espacios no cristianos que observan el perdón como modalidad de desactivación y manejo de conflictos (Derrida 2006). Especialmente las comisiones de verdad y reconciliación, desde fines del siglo XX en adelante, entran en esta lógica (Kastner 2012, 2015).

\subsection{FORMA SOCIO-INTEGRATIVA POSITIVA}

En la forma socio-integrativa positiva de memoria, la historia es fuente de solidaridad e integración social en términos de la continuidad de una tradición que debe constituir la base para la reproducción presente de la identidad social del colectivo. Fechas simbólicas de batallas, nacimientos o muertes de personajes, fundaciones, pactos, leyes, armisticios, etc. (celebra- 
das con mayor énfasis en números redondos, como el bicentenario) se suman a sitios o espacios en los que habrían ocurrido eventos clave para la nación, y a ellos se les suma además una inmensa carga de cultura material conmemorativa (monumentos, calles, plazas, parques, escuelas, edificios, monedas, etc.) que puebla el paisaje urbano. La política oficial de conmemoración de los Estados nacionales, expresada además en programas de enseñanza, supone un trabajo de extrema selectividad en relación con el pasado. Como tal, constituye un esfuerzo pragmático de construcción y reproducción de la memoria colectiva, la tradición y la conciencia nacional.

Tales procesos de construcción social mediante la memoria han sido objeto de análisis sociológico. Estos se han realizado desde vertientes de orden más normativista, así como de otras asociadas con la historiografía crítica y el vínculo entre sociología histórica y del conflicto. La versión normativa de la conmemoración acentúa el potencial de integración normativa y de generación de solidaridad de los procesos de construcción de la memoria y la representación colectiva (Durkheim 2003; Halbwachs 1997). La versión de la sociología del conflicto se esfuerza por develar formas de cohesión social y legitimación simbólico-ideológica de relaciones de poder y dominación en los procesos de construcción nacional de la memoria colectiva (Hobsbawm \& Ranger 1983; Anderson 1983). En todo caso, ambas sociologías - la normativa y la del conflicto- conciben la memoria colectiva como un objeto de conmemoración oficial y ritual que no es ni la historia misma ni el mero pasado, sino que un proyecto nacional apoyado en la selectividad de eventos cargados pragmáticamente de significado.

Una modalidad distinta de la forma socio-integrativa positiva de la memoria se desarrolla actualmente al trasladar la mirada desde los proyectos nacionales hacia las formas de construcción de una sociedad mundial. En estos casos, se trata de la integración normativa de la sociedad mundial sobre la base del horizonte de derechos humanos como marco normativo del derecho internacional público o de un derecho transnacional. Esta forma socio-integrativa positiva incluye, por ejemplo, la dimensión propositiva de la perspectiva de Habermas centrada en la constitucionalización de la sociedad mundial (Habermas 2008). Si bien, en su origen, la perspectiva habermasiana puede ser clasificada en la forma socio-integrativa negativa, en la que la memoria del holocausto constituye aquello que debe ser a toda costa evitado en la formación política de presente y futuro, la propuesta positiva de integración se basa en la actualización permanente de los derechos hu- 
manos como memoria normativa constitucionalizada de la sociedad mundial. Esta estructura tendría un carácter supranacional (regional o continental) y se centraría en el mantenimiento de la paz y la implementación de los derechos humanos. Adicionalmente, ejercería una supervisión sobre espacios de governance transnacional que carecen de modalidades de legitimación democrática (Habermas 2008) y se conectaría finalmente con los Estados nacionales democráticos en forma de flujos legitimatorios de procesos decisionales. Con ello, la sociedad mundial quedaría vinculada en un horizonte normativo de integración global, tanto desde el nivel supranacional como desde el nacional.

Una propuesta similar a esta es la de Robert Fine (2012). En este caso, la memoria se construye sobre la noción de 'crímenes contra la humanidad' desarrollada en los juicios de Núremberg. El componente socio-integrativo positivo se presenta aquí en forma de un argumento político que promueve una cultura de derechos humanos a la que se puede recurrir "cuando enfrentamos nuevos problemas de convivencia como colectividad humana" (Fine 2012: 39). El derecho internacional de la sociedad mundial debe, por tanto, evitar la naturalización de los derechos humanos, pues ello introduciría diferencias en niveles de humanidad entre regiones del mundo -por ejemplo, en la estratificación rawlsiana entre estados liberales, decentes y outlaw (Rawls 2000) - y atentaría contra la construcción política de la comunidad humana.

En síntesis, las formas de la memoria en el siglo XX han enfatizado su dimensión negativa (tanto cognitiva como socio-integrativa). De una parte, se trata de aprender de la tragedia histórica para no repetirla (forma cognitiva), de la otra, se trata de integrarla, como tal, en la conciencia colectiva, de cara a la construcción de una integración social auto-crítica y reflexiva (forma socio-integrativa). Esto no quiere decir, en absoluto, que las otras formas de relación entre historia y política desaparezcan. En el siglo $\mathrm{XX}$, la forma cognitiva positiva pierde relevancia pública como historia magistra vitae y se transforma en discusión de expertos. En el caso de la forma socio-integrativa positiva, ella puede permanecer en las tradiciones nacionales o incluso reactivarse ahora en un nivel global como construcción de una tradición de derechos humanos. 
2. El caso del Museo de la Memoria y los Derechos Humanos, CHILE

El aparato conceptual desarrollado arriba puede ser visto en acción en un caso empírico: la controversia en torno al MMDH. Esta controversia ha tenido lugar desde el propio anuncio de la construcción del Museo en 2007, llegó a un punto más alto en 2012 y continúa hasta la actualidad. En esta sección reconstruyo esa controversia empíricamente y la pongo en relación con las formas abstractas de la memoria identificadas en la sección anterior.

\subsection{Metodología}

La investigación empírica sobre la controversia en torno al MMDH ha sido realizada sobre archivos de prensa publicados entre los años 2007 (año del anuncio de la creación del Museo) y 2016 (año de inicio de redacción del presente artículo). En tanto lo que interesa a este trabajo es la controversia pública sobre el tema, es decir, lo que diversos actores proponen públicamente sobre la labor del Museo (no lo que privadamente opinan, por ejemplo, en situación de entrevista), los archivos de prensa constituyen el material central.

Las fuentes utilizadas son los periódicos chilenos El Mercurio (108), La Tercera (43), La Segunda (13), El Mostrador (21) y The Clinic (15), tanto en sus versiones electrónicas como impresas (cuando existen). Puesto que el debate público se llevó a cabo principalmente a través del diario El Mercurio, este tiene una representación mayor en la muestra. En total se revisaron 200 artículos de prensa seleccionados bajo conceptos de búsqueda adecuados a las hipótesis, tales como memoria, historia, controversia, debate y derechos humanos, relacionando siempre estos conceptos con el término central 'Museo de la Memoria', el que debía aparecer relacionado con los otros conceptos indicados. Esto es, no se seleccionó, por ejemplo, 'derechos humanos' de forma aislada, sino 'derechos humanos' y 'Museo de la Memoria'. En su mayoría, estos artículos de prensa son cartas al director, algunas de representantes de instituciones directamente involucradas $(\mathrm{Mu}-$ seo de la Memoria, Dibam), y otros de actores individuales (generalmente académicos, columnistas o políticos). También se incluyeron en la muestra artículos informativos sobre el Museo (anuncios de su creación, noticias de 
su inauguración y de sus aniversarios) y otros reportajes o entrevistas que apuntaban a las controversias identificadas.

Adicionalmente, con el objeto de complementación de información, se revisaron otros portales electrónicos (radio Biobio, Cambio 21, Radio Universidad de Chile) en los que hubiese información no considerada en las fuentes mencionadas más arriba. El análisis se estructuró sobre una base temporal para identificar los momentos de intensidad de la controversia y matrices temáticas para identificar los contenidos de las formas de la memoria.

\subsection{LA FORMA DE LA CONTROVERSIA}

Sostengo que el MMDH adopta una 'forma socio-integrativa negativa', donde el pasado dictatorial de Chile entre 1973 y 1990 es considerado como fuente de autorreflexión crítica: mantener viva la memoria de $Y$ (violación de los derechos humanos) para una ética común de un 'Nunca Más $Y$ ' (Palma 2007; Brodsky 2012; Museo de la Memoria 2016). Pero que, a la vez, se abre hacia una 'forma socio-integrativa positiva' que apunta a la creación de una ética común del respeto de los derechos humanos.

Las críticas más elocuentes al MMDH pueden ser diferenciadas en tres tipos, cada uno de ellos codificable respectivamente en términos de una de las formas abstractas identificadas en la sección anterior:

a) Existe una controversia entre la propuesta socio-integrativa negativa del $\mathrm{Mu}$ seo y una crítica en términos de 'neutralización de la memoria' que luego da paso a una primera formulación de 'crítica contextualizadora' (necesidad de antecedentes que expliquen las violaciones a los derechos humanos en el período 1973-1990). Esta primera formulación de la crítica contextualizadora, se asocia con la forma cognitiva negativa de la memoria en lo que denomino primera fase de la controversia (2007-2010).

b) Una segunda fase de la controversia, que adquiere su momento más alto en 2012, se produce en torno a una segunda formulación, más sofisticada, de la crítica contextualizadora y la función pedagógica del Museo. La crítica supone que éste debiese 'contextualizar' la violación de los derechos humanos para cumplir verdaderamente el rol pedagógico que se le encomienda. 'Contextualizar' quiere decir, en este caso, construir una explicación justificatoria de las razones que conducen al quiebre de 1973 y las violaciones a los derechos humanos producidas entre 1973 y 1990. Esta crítica se expresa en los términos de la forma abstracta cognitivo-negativa de la relación entre historia y memoria, es decir, estableciendo que, para que se pueda aprender 
de la historia y tomarla como 'advertencia' es necesario conocer la relación causal entre antecedentes y consecuentes (violencia política en el pasado que lleva a la dictadura). Esta segunda fase debate también sobre la distinción entre memoria e historia.

c) En la tercera fase (2012-2016), la controversia versa fundamentalmente en torno a la inclusión de grupos que observan sus derechos humanos conculcados y que, mediante la crítica al Museo, formulan la expectativa de ser considerados en su espacio y actividades. Esto abre un período en el que, junto con la forma socio-integrativa negativa que se mantiene como fundamental en la propuesta del Museo, emerge también una forma socio-integrativa positiva fundada en el despliegue de una cultura de derechos humanos y valores democráticos.

En lo sucesivo, observo más detenidamente estas fases de la controversia en sus detalles y variaciones.

\subsection{PRIMERA FASE 2007-2010: REFLEXIÓN, NEUTRALIZACIÓN Y CON- TEXTUALIZACIÓN}

Una de las primeras construcciones públicas del $\mathrm{MMDH}$, donde se identifican los fundamentos de la forma socio-integrativa negativa a la que responde, es la que se expresa en el siguiente pasaje de la directora de Dibam (una de las instituciones a cargo en el proceso de gestación del Museo) en 2007, Nivia Palma. La propuesta de Museo debe rescatar:

El tema de las violaciones de los derechos humanos en el país, pero en la línea del aprendizaje y del respeto a la vida, generando conciencia que ojalá no vuelvan a ocurrir en situaciones como las anteriores. No queremos que el público salga desmoralizado y con la idea de no volver más. El museo no dimensionará sólo la parte dolorosa de la historia, sino también otros valores, como la reflexión. (Palma 2007).

La formulación deja ver elementos clave de una forma socio-integrativa negativa, estos son: la apropiación crítica de la historia y la reflexión sobre ella. Muestra también que la forma socio-integrativa negativa incluye también un componente cognitivo, tal es el aprendizaje del pasado; pero en tanto este aprendizaje es concebido en su relación con la integración social y no solo como advertencia (el aprendizaje debe 'generar conciencia'), la posición adopta la tonalidad general que he identificado como la forma socio-inte- 
grativa negativa de la memoria. No se trata, por tanto, solo de un mero aprendizaje, sino de un aprendizaje normativo.

Ya tempranamente, ante este anuncio, aparecen voces críticas desde posiciones políticas de una derecha que hoy continúa apoyando a la dictadura militar en su conjunto. Esta crítica conduce a una neutralización de la memoria; no en el sentido de una negación explícita a las violaciones de los derechos humanos durante la dictadura, pero sí en cuanto a su valoración y los parámetros que se emplean para establecer tal valoración. La neutralización se expresa en la forma de contra-trasgresiones. Por ejemplo: “¿Estarán en ese museo los sacos con que Castro envió metralletas a la UP? ¿Se mostrará el armamento ingresado por Carrizal Bajo y el estado de los autos de la comitiva del presidente Pinochet después del intento de asesinato?" (Rojas 2007). En este caso, las violaciones de derechos humanos se hacen simétricas con acciones particulares. La controversia queda paralizada, salvo como incremento del potencial de conflicto. La búsqueda de simetría no alcanza el estatuto de 'contextualización', pues para ello falta la argumentación; tiene un sentido más bien retórico, cuya fuerza simbólica, de todos modos, no puede desconocerse.

En un tono más argumentativo, la neutralización de la memoria también puede producirse buscando eliminar la excepcionalidad del período 1973-1990:

No me parece atinada la idea de crear un museo cuya misión será mantener abierto el tema de los derechos humanos. No se trata de olvidar lo sucedido ni mucho menos. Lo correcto sería que los episodios de violencia política ocurridos en nuestra historia tuvieran un espacio en el Museo Histórico Nacional, quedando así integrados. (Clavel 2007).

En este caso tampoco hay una pretensión de contextualización de las violaciones a los derechos humanos; más bien se renuncia a atribuir un estatuto especial al hecho histórico, situando su valoración a nivel de otros episodios de violencia política ocurridos en la historia de Chile.

Una crítica distinta al proyecto de un $\mathrm{MMDH}$ es la que aparece en el mismo año 2007 desde una posición política liberal. Se trata ante todo de una crítica a la unidad entre memoria y Estado, formulada por Sol Serrano:

El concepto de memoria utilizado por el Estado es otra cosa y requiere una reflexión de aquello que le compete al Estado. Habría que definir cuál memoria y de quiénes. Si se trata de mostrar la violencia política como una pedagogía ciu- 
dadana, entonces habría que tratarla a lo largo de toda la historia o desde algún periodo en el cual se considere que había derechos que se conculcaron. (Serrano 2007).

La posición busca explícitamente evitar el riesgo de la neutralización: "el empate de los muertos, la equivalencia de crímenes, la nivelación de la violencia" (Serrano 2007) y poner el énfasis en el pluralismo que los organismos públicos deben defender. Ello no implica sumar versiones, sino mostrar los quiebres históricos del pluralismo. Vista de esta forma, la argumentación se sostiene en la que he denominado forma cognitiva negativa de la memoria, es decir, aquella que entiende el pasado como advertencia. La historia, en este caso, es un escenario de cuyas catástrofes (independiente de su tipo) se puede aprender como fuente de 'pedagogía ciudadana'. Sin embargo, en tanto el MMDH se concibe a sí mismo en términos socio-integrativos negativos a partir de un período definido (1973-1990), la crítica supone igualmente una forma de neutralización de lo acontecido en él. Cuando el parámetro de valoración es la 'falta de pluralismo' en Chile, el período no sería simbólicamente excepcional como para distinguirlo de otros y debiera quedar incluido en un horizonte mayor, en el que no se ponga énfasis directamente en las violaciones sistemáticas a derechos humanos por parte del Estado, sino en la violencia política que interrumpe la pluralidad y diversidad de la sociedad.

La idea de que el período en cuestión quede incluido en un horizonte temporal mayor está, a mi juicio, en la raíz del debate público acerca de la contextualización histórica de las violaciones a los derechos humanos durante la dictadura militar que el Museo tendría que hacer. Este debate se produce entre posiciones políticas opuestas, pero más moderadas (centroderecha y centro-izquierda) y emerge con notoriedad pública por primera vez a inicios de 2010, en el mismo seno institucional del MMDH, esto es, entre los directores del Museo nombrados durante el primer gobierno de Bachelet (entre otros, Arturo Fontaine, Agustín Squella, Milan Ivelic, María Luisa Sepúlveda, Carlos Peña, Fernando Montes). La idea de "contextualización" (formulada por Ivelic) supone "la necesidad de 'ampliar' la muestra para que no sea sólo respecto de los hechos ocurridos entre 1973 y 1990, sino que también se aborde el 'contexto histórico' del gobierno de Salvador Allende y el golpe militar de septiembre de 1973" (El Mercurio 2010). Bajo la exigencia de contexto histórico, la memoria adopta una forma cognitiva negativa, como advertencia: si acontece X, entonces Y. En tanto el Museo 
se sostiene en su forma socio-integrativa negativa, la advertencia no es necesaria, pues Y (el golpe, la violación de derechos humanos) debe ser ya, por principio, inaceptable: su ocurrencia en el pasado determina su inaceptabilidad futura y determina también esta negación como fuente de integración. En tal sentido, María Luisa Sepúlveda (en el momento presidenta del directorio), ratifica la función socio-integrativa negativa en los siguientes términos: "dar cuenta de los hechos sucedidos entre 1973 y 1990 y hacer una reflexión ética del atentado a la dignidad que ello significó" (Sepúlveda 2010). La posición contiene ciertamente un componente cognitivo ('dar cuenta de los hechos'), pero el integrativo ('reflexión ética') domina normativamente la formulación.

En los días previos y posteriores a la inauguración del Museo, el debate adquirió un tono más confrontacional. Así, el 11 de enero de 2010, "adulteración histórica inescrupulosa" (Farías 2010), "disfraz de historia" (Bazán 2010), "historia indiscutiblemente sesgada e incompleta" (Retamal 2010), fueron algunas de las voces críticas al Museo; mientras otras buscaban combinar el sentido socio-integrativo negativo con la necesidad cognitiva negativa de contextualización: "Las nuevas generaciones [...] deben hacer esfuerzos por no repetir errores, y para eso es necesario no olvidar lo que ocurrió [...] eché de menos que no se contextualizara la época en que se produjo este capítulo de nuestra historia, que diera cuenta de las cosas que llevaron al caos" (Monckeberg 2010). También, en un tono similar: "considerar el contexto histórico de actos intrínsecamente malos como la desaparición o tortura de personas no significa en modo alguno justificarlos moralmente; por el contrario, contribuye a tomar conciencia de sus causas y a conocerlos más completamente, de modo que sea posible evitar en forma efectiva que ellos vuelvan a ocurrir en nuestro país" (Ugarte 2010).

En términos teóricos, el foco de la controversia se centra, por tanto, en si la forma socio-integrativa negativa de la memoria requiere o no de una estructura de causalidad que explique el evento traumático que aporta unidad moral por la vía de su rechazo. En tanto esta forma valora el evento particular de manera negativa por principio (inaceptabilidad de la violación de derechos humanos), su contextualización no se hace necesaria. Aun cuando la contextualización pueda realizarse y aun cuando ella mostrase eventualmente la irracionalidad de la acción cuya negación se toma como fuente de unidad moral, ello no agregaría nada decisivo a la forma socio-integrativa negativa: el conocimiento de hechos previos no determina ni afecta la conciencia autocrítica que surge del evento negativo. 


\subsection{SEGUNDA FASE 2012: CONTEXTUALIZACIÓN, HISTORIA Y MEMORIA}

La segunda fase de la disputa en torno el MMDH se produce en el año 2012 (durante el gobierno de Sebastián Piñera) y retoma el tema de la contextualización con mayor sofisticación, también entre posiciones de centro izquierda y centro derecha, introduciendo la distinción entre historia y memoria.

El debate sobre la contextualización se centra en la 'desinformación' (aspecto cognitivo) que un Museo acotado al período 1973-1990 produciría en nuevas generaciones, lo que haría necesario un Museo de la Memoria II (Rillón 2012). Frente a ello, el entonces director del MMDH Ricardo Brodsky (2012) sostiene la responsabilidad pedagógica del Museo para con esas nuevas generaciones "con el fin de fortalecer los valores democráticos y el Nunca Más". En este punto, la controversia incluye elementos de las distintas formas de la memoria expuestas en la sección anterior. Por un lado, se apela a un sentido cognitivo negativo (contextualización, pedagogía como información de antecedentes) y, por otro, a un sentido socio-integrativo, tanto negativo (representado en el 'Nunca Más') como positivo (representado en la importancia atribuida a valores democráticos). La controversia adquiere un punto crítico con la carta de Magdalena Krebs (entonces directora de Dibam). En ella, Krebs acusaba el carácter limitado de la función pedagógica del museo, en tanto este no daría cuenta del rol relevante de la violencia política en los años previos al golpe (Krebs 2012a). Krebs enfatiza ahí un concepto de 'pedagogía' informativo (explicación de antecedentes), mientras que Brodsky pone el acento en la dimensión normativa del concepto (formación valórica). En tal sentido, la posición de Krebs adquiere un tono cercano a la forma cognitiva negativa, en la que el pasado como advertencia juega un rol clave: "sería una gran contribución que el museo explicara los hechos anteriores al golpe, pues pondría una nota de atención sobre los límites, la necesidad de cuidar las formas y los procedimientos democráticos" (Krebs 2012a).

La idea de contextualización es apoyada, desde una posición más confrontacional, por el historiador Sergio Villalobos. Aquí, la historia es concebida como descripción de secuencias: "Desde el punto de vista de la historia, la existencia del museo representa el deseo de falsificar el pasado, en cuanto se enfoca en un acontecimiento singular, separado del resto de nuestra historia y, por lo tanto, incomprensible" (Villalobos 2012a). Bajo 
esta perspectiva, la historia siempre debiera ser 'historia total', es decir, una historia de los antecedentes de los antecedentes. Como lo sostiene Víctor Farías (2012a), quien suscribe las posiciones de Villalobos y Krebs: "[un museo] debe ser racionalmente explicatorio de una totalidad histórica o cultural". Estas formulaciones, y especialmente la de Krebs, son entendidas en el debate en forma de explicaciones justificatorias de las violaciones a los derechos humanos (Palet 2012, Parada 2012). En rechazo de esto, Krebs adopta algunos elementos de la forma socio-integrativa negativa:

Concuerdo plenamente en que las circunstancias no justifican las violaciones a los Derechos Humanos y reconozco como un valor del Museo de la Memoria que éste transmita esa cruda realidad que fue negada durante muchos años. Mi planteamiento sólo apuntaba a que, si como país queremos que ello no se repita, debemos estar abiertos a la reflexión. (Krebs 2012b).

El concepto de reflexión, en todo caso, no es introducido en el sentido de 'conciencia crítica' que adopta en la forma socio-integrativa negativa, sino más bien en su forma cognitiva: la aspiración de Krebs sigue siendo la de un museo que aporte un relato de 'alta complejidad' donde se dé cuenta de los antecedentes.

En el curso del debate, el propio directorio del Museo interviene para establecer que su misión no es historiográfica ni jurídica: no es contextualizar ni imputar responsabilidad, sino promover "un diálogo reflexivo acerca del respeto a los derechos humanos" (Sepúlveda, Rojas, Fontaine, Gómez, Ivelic, Montes, Nash, Palet, Peña, Platovsky, Romero, Scantlebury, Squella y Tohá 2012). En este sentido, el Museo también muestra una forma sociointegrativa positiva orientada a la promoción de la cultura de derechos humanos, y no solo una forma socio-integrativa negativa representada en el 'Nunca Más'. Esta posición socio-integrativa positiva también había sido formulada por Sebastián Piñera (entonces presidente) en su primera visita al Museo en abril de 2012: "mirar hacia adelante y crear en Chile una cultura de tolerancia y de respeto a los Derechos Humanos, porque esa es la mejor manera de protegernos de cualquier intento, hoy día o en el futuro, de atentar contra los derechos de las personas o contra la dignidad de las personas" (Piñera 2012).

Paralelo a esto, el debate muestra una orientación a discutir la distinción entre historia y memoria. La memoria tendría una función pedagógica normativa, en especial hacia aquellos "que no vivieron el drama" (VieraGallo 2012); mientras que la historia tendría una función pedagógica expli- 
cativa en tanto "indaga las causas de los acontecimientos recurriendo a las fuentes que contribuyen a su explicación" (Viera-Gallo 2012). En subsidio de la posición normativa, Agustín Squella (2012a) señala que el Museo "no fue concebido para liberar de su trabajo a los historiadores, a los sociólogos, a los cientistas políticos ni a cualquier otro colectivo interesado en aclarar (o más bien en interpretar) un momento tan grave de nuestra historia patria". Para esta posición, la construcción de memoria que hace el Museo constituye una selección con fines normativos (o socio-integrativos), mientras que la historia adopta un carácter científico. Farías (2012b) tiende a disolver esta distinción entre memoria e historia, al sostener que el acto de 'mostrar' en el MMDH "supone el esencial aporte de la historiografía fundada", pues de otro modo "no se mantienen vivas y alertas las conciencias, sino que se las manipula". En un sentido similar, Villalobos subordina la memoria a la historia entendida como ciencia: "Cualquier tipo de memoria, sobre cualquier hecho ocurrido en el pasado, es recogido por la historia, una disciplina que posee teoría y método y no puede ser utilizada de manera desordenada" (Villalobos 2012b). Squella (2012b), en tanto, repone la función socio-integrativa al entender que 'mostrar' es la forma selectiva de la memoria y del Museo de la Memoria.

Esta fase se estructura, entonces, del modo siguiente: si la memoria es entendida como subordinada a la historia, el Museo requiere de contextualización para explicar las violaciones a los derechos humanos (forma cognitiva negativa) -al Museo se le atribuye en este caso una función pedagógica en el sentido de información sobre los antecedentes. Si, por el contrario, la memoria es entendida de manera independiente de la historia, entonces las violaciones a derechos humanos se asumen como punto de partida para generar una reflexión autocrítica que conduzca al 'Nunca Más Y' (forma socio-integrativa negativa) y que promueva una cultura de derechos humanos en el país (forma socio-integrativa positiva).

\subsection{TERCERA FASE 2012-2016: EXTENSIÓN HACIA LA PERSPECTIVA SOCIO- INTEGRATIVA POSITIVA}

El fundamento de la función del MMDH está en la forma socio-integrativa negativa de la memoria. Esto se mantiene hasta hoy. Sin embargo, desde el seno de la controversia sobre la contextualización, surge en 2012 la significación pública del Museo como forma socio-integrativa positiva que enfa- 
tiza la relevancia de los derechos humanos como un sustrato cultural de carácter universal. Esta perspectiva va más allá de las violaciones a los derechos humanos durante la dictadura, es decir, agrega al 'Nunca Más Y' (forma socio-integrativa negativa) la aspiración de un sentido general de derechos humanos que incluye a otros actores cuyos derechos son actualmente conculcados. En este sentido, las posiciones políticas que se enfrentan coinciden en la valoración socio-integrativa del tema, pero se distancian en cuanto a la variedad de colectivos representados o incluidos en el mensaje del Museo.

Como lo he mostrado, la forma socio-integrativa positiva está ya presente en un nivel secundario, en la autodescripción del propio Museo desde su origen: se observa en el "aprendizaje y respeto a la vida" (Palma 2007) y en el "fortalecimiento de valores democráticos" (Brodsky 2012). No obstante, una primera evidencia pública concreta de expectativas y de acciones dirigidas a la extensión de la función socio-integrativa positiva se produce en la misma inauguración del Museo, cuando una activista mapuche (identificada como hermana del comunero mapuche Matías Catrileo, muerto en enfrentamiento con Carabineros en la Región de la Araucanía), sostiene que “En Chile se violan los derechos humanos!” (El Mostrador 2010). La acción constituye una crítica a la identificación que el Museo hace entre violación a los derechos humanos y dictadura -identificación vista como restrictiva. No obstante, la crítica o la denuncia muestran que la pretensión del Museo de contribuir a una cultura de derechos humanos tiene eco en otros grupos que también describen su situación actual como limitación de derechos. La inquietud manifestada en la inauguración es recogida por el Museo en 2011 con el proyecto de apertura de un espacio mapuche que dé cuenta el impacto en "la organización social y humanitaria del pueblo mapuche" (Brodsky 2011) durante la dictadura. Esto incluye, no solo a las víctimas de la violencia sino también la política de tierras durante el período. En lo sucesivo, estas actividades han incluido, desde muestras fotográficas hasta la audioguía en mapudungun (Cambio 21 2013) y el apoyo a diferentes actividades artísticas (Municipalidad de Melipilla 2015).

Una segunda evidencia de la ampliación del foco de derechos humanos en términos de una perspectiva socio-integrativa positiva se activa también a partir de una crítica, la de Francisco Casas (2013) y Pedro Lemebel (2013), en torno al desconocimiento, por parte del Museo, de las obras de 'Las Yeguas del Apocalipsis' (conjunto de performances de Lemebel y Casas que reflexionan sobre el cuerpo como espacio político). Como en el 
caso anterior, la crítica presupone una expectativa de inclusión de la perspectiva homosexual en el enfoque del Museo, desde un grupo que observa sus derechos limitados. El Museo también comienza a hacerse cargo de esta crítica por medio del apoyo a distintas iniciativas de discusión y artísticas (exposiciones de video, performances y películas) de grupos homosexuales. No obstante, el acto más evidente de inclusión de esta perspectiva tiene lugar en enero de 2016, cuando en el aniversario del Museo se inaugura en sus instalaciones la muestra 'Arder' de Pedro Lemebel que incluye registros fotográficos y audiovisuales de las distintas performances realizadas por el artista, cuya ausencia hacían notar en 2013 los propios Casas y Lemebel (biobiochile.cl 2016).

La ampliación y consolidación de la perspectiva socio-integrativa positiva en el MMDH, expresada en la formación de una cultura de valores democráticos y derechos humanos, es aún débil. Sin embargo, la extensión a temáticas mapuches y homosexuales muestra que la inclusión de un enfoque ampliado de derechos humanos (que, en todo caso, está presente desde la concepción del Museo) tiene un correlato en actividades concretas que son incorporadas luego de reflexiones críticas de tipo público. Es decir, las expectativas que abre el Museo para la ampliación de la perspectiva de derechos humanos adquieren resonancia pública en grupos que observan sus derechos limitados de uno u otro modo y que entienden al Museo como un espacio que contribuye a su estabilización.

\section{CONCLUSIONES}

Dos hipótesis relacionadas guiaron este artículo: a) que las disputas de la memoria pueden ser entendidas como controversias entre distintas formas sociales abstractas de construir la relación entre historia y política por medio de la memoria, y b) que la controversia en torno al $\mathrm{MMDH}$ es, en último término, una controversia entre distintas formas abstractas de la memoria.

Una primera conclusión, a partir de esta investigación teórico-empírica, es que las distintas formas de la memoria se organizan según intereses políticamente definidos que pueden reconstruir la historia en sentidos opuestos generando controversia. Las posibilidades son cuatro: a) cuando posiciones ideológicas de derecha e izquierda colisionan, la controversia puede neutralizar la memoria por medio de una simetrización o 'equivalencia de crímenes' - primera fase de controversia del MMDH; b) cuando las 
posiciones políticas son más moderadas, la controversia se produce entre un énfasis que denomino cognitivo negativo (la historia como advertencia) y otro que llamé socio-integrativo negativo (la historia como reflexión autocrítica) -en nuestro caso, especialmente la segunda fase de la controversia; c) cuando las posiciones políticas coinciden en la valoración socio-integrativa del evento en cuestión, la controversia puede producir una complementariedad entre el componente cognitivo y la forma socio-integrativa negativa de la memoria: la historia puede verse como advertencia, pero se debe subordinar ello a la reflexión autocrítica (el MMDH informa los antecedentes que muestran las violaciones de derechos humanos y las vincula a un sentido normativo autocrítico representado en el 'Nunca Más'); y d) cuando las posiciones políticas coinciden en la valoración socio-integrativa del evento, pero se distancian en cuanto a la extensión de la representación, la controversia se produce a nivel socio-integrativo entre una forma negativa ('Nunca Más Y') y una forma positiva (afirmación de cultura de derechos humanos y valores democráticos para la inclusión de diversidad). En este último caso, la complementariedad también es posible, como se muestra en la tercera fase de la controversia en relación con el caso mapuche y de la diversidad sexual. En todos los casos, la memoria articula la relación entre aspiración política en el presente e interpretación de la historia. En tal sentido, la memoria es una reconstrucción de la historia (de un evento, de un período) formulada políticamente desde el presente.

Una segunda conclusión es que la crítica es una práctica fundamental para la distinción entre formas de la memoria. Todas las controversias producidas emergen a partir de la crítica pública que, desde una posición política, se realiza a otra posición que queda configurada como contraria. La crítica es el fundamento operativo de la controversia y, al serlo, es también la operación que permite la emergencia y diferenciación entre distintas formas de la memoria. En tal sentido, las formas de la memoria no existen $a$ priori, sino que emergen, se distinguen y se consolidan en la controversia. Como el análisis del caso del MMDH de Chile lo muestra, el elemento que está en la base de la dinámica de la controversia es la crítica: primero, la crítica a la no consideración de actos que se juzgan equivalentes y la crítica a la unidad entre memoria y Estado en la primera fase; segundo, la crítica a la necesidad de contextualización en la segunda fase; y tercero, la crítica a una representación restringida de violaciones a derechos humanos y que promueve su pluralización. En todos los casos, la crítica motiva y produce la dinámica de las formas de la memoria. 
Una tercera conclusión concierne a la trayectoria de la controversia sobre el MMDH de Chile. La controversia puede ser efectivamente representada en las formas de la memoria. En la primera fase (anuncio de la creación del Museo), la forma socio-integrativa negativa que adopta el Museo, representada en el 'Nunca Más' la violación sistemática de derechos humanos, se cuestiona por medio de la neutralización de la memoria mediante la fórmula de 'equivalencia de crímenes'. Se pone en duda aquí la propia existencia del Museo. En la segunda fase, ya aceptada la creación del mismo, se pone en duda su modelo de memoria por medio de la fórmula de la 'contextualización'. Y en la tercera fase, se cuestiona la restricción de su representación (forma socio-integrativa negativa centrada en los crímenes de la dictadura) por medio de una demanda de extensión de su foco a otros grupos sistemáticamente vulnerados en sus derechos. Dos posiciones analíticas quedan excluidas en esta trayectoria. Primero, la forma cognitiva positiva (historia como modelo de acción) no aparece en la controversia. En el modelo teórico he sostenido que esta forma de la memoria pierde relevancia en el siglo XX y queda reducida a una discusión de expertos. Una posibilidad de identificar esta forma podría estar en las visiones que sostienen la "ejemplaridad" de la transición chilena a la democracia (Cañas 1997) y en la discusión de ella (Garretón 2004), no obstante, esta disputa no surge en los materiales revisados sobre la controversia pública. Segundo, la forma socio-integrativa positiva tiene una versión nacional (centrada en la conmemoración ritual de gestas y hechos heroicos) y una versión universalista (centrada en la formación de una tradición de derechos humanos). En el modelo teórico sostuve que la versión nacional es propia del siglo XIX y primera mitad del $\mathrm{XX}$, y que la versión universalista comienza a surgir en la segunda mitad del XX. En la controversia sobre el Museo de la Memoria, la primera versión no emerge en el plano público (una posibilidad de identificarla es por medio de la idea de 'gesta de 1973' en posiciones de derecha extrema); la segunda versión, sin embargo, domina la controversia en torno al $\mathrm{MMDH}$ en su tercera fase. Ello puede inducir a pensar en un proceso relativamente exitoso del foco extendido de derechos humanos y valores democráticos que el Museo plantea desde su origen. Aun cuando grupos con derechos conculcados demanden la inclusión por medio de una crítica al Museo, es la misma existencia de él la que produce la expectativa de inclusión y, por tanto, la crítica y demanda por inclusión. Esta observación, sin embargo, solo podrá constatarse a futuro en investigaciones que determinen el carácter y alcance 
de ese foco extendido con base en una forma socio-integrativa positiva de la memoria.

Una conclusión final dice relación con los límites entre las fases identificadas. Puesto que la controversia adquiere momentum, esta no puede concebirse de manera continua. La primera fase (2007-2011) se sostiene en un nivel relativamente bajo, desde el anuncio de creación del Museo hasta su inauguración, con énfasis en su inicio y su culminación. La segunda fase adquiere nivel crítico en 2012, pero se sostiene esporádicamente en especial en los aniversarios del Museo (por ejemplo, Villalobos 2014). La tercera fase (2012-2016) tiene un nivel de intensidad bajo también con peaks en los aniversarios del Museo, aunque la idea de un foco extendido de los derechos humanos y de los valores democráticos está presente desde el inicio. En este sentido, las transiciones entre fases no son precisas; presentan más bien períodos de incubación, momentos críticos y otros de latencia. Ello también habla en favor de la relevancia del presente político para la construcción (o reconstrucción) de la memoria y para la emergencia de sus controversias.

En síntesis, la controversia en torno al MMDH en Chile muestra que la crítica de posiciones políticas en relación a la historia genera 'formas de memoria' con énfasis cognitivos y socio-integrativos, que esas formas se conectan a expectativas de inclusión en esquemas de representación institucional, y que los énfasis de la controversia cambian en el tiempo de acuerdo con las expectativas que los mismos esquemas institucionales y las condiciones políticas de una época abren a los actores sociales. En tanto las formas de la memoria surgen de la praxis de la crítica, no es posible anticipar que cada conflicto de memoria pueda ser interpretado bajo las categorías desarrolladas aquí. Sin embargo, en tanto formas sociales abstractas en el sentido de Simmel, ellas pueden ser aplicables a otros contextos con características histórico-institucionales similares. $\mathrm{M}$

\section{REFERENCIAS}

Anderson, B. (1983). Imagined Communities: Reflections on the Origin and Spread of Nationalism. London: Verso.

Assmann, A. (2006). Memory, Individual and collective. In R. Goodin \& C. Tilly (Eds.), The Oxford Handbook of Contextual Political Analysis (pp. 210-224) Oxford: Oxford University Press. 
Hacia una reconstrucción de los conflictos de la memoria. El caso del Museo de la Memoria y los

Basaure, M. (2001). Prudencia y ciencia en el Leviathan de Thomas Hobbes. Crítica interna al rechazo de la prudencia como conocimiento válido para la política. Revista Jurídica 3, 4255.

Basaure, M. (2013). From the History of the Peloponnesian War to Leviathan Hobbes and the Knowledge and Wisdom for Peace. New Centennial Review, 13(1), 71-92.

Basaure, M. (2014). Aprender críticamente de la historia. De la posibilidad de un diálogo al interior de la controversia en torno al Museo de la Memoria y los Derechos Humanos. In G. Bustamante \& A. Estefane (Eds.), La agonía de la convivencia (pp. 171-194). Santiago de Chile: RIL.

Bazán, J. (2010). Museo de la Memoria. El Mercurio, 12 de enero 2010.

Biobiochile.cl (2016). 'Arder': la muestra de Pedro Lemebel se inaugurará en el Museo de la Memoria. Biobiochile.cl, 14 de enero 2016. Disponible en: http://www.biobiochile.cl/noticias/2016/01/14/arder-la-muestra-que-pedro-lemebelse-inaugurara-en-el-museo-de-la-memoria.shtml [25 de diciembre 2016].

Brodsky, R. (2011). En 'Museo de la Memoria y los Derechos Humanos abre sus puertas a los temas mapuches'. El Mostrador, 23 de mayo 2011.

Brodsky, R. (2012). Museo de la Memoria. El Mercurio, 21 de junio 2012.

Brumlik, M. (2004). Aus Katastrophen lernen? Grundlagen zeitgeschichtlicher Bildung in menschenrechtlicher Absicht. Berlin: Philo \& PhiloFineArts, Europäische Verlagsanstalt.

Brunkhorst, H. (2005). Solidarity. Cambridge, Mass.: The MIT Press.

Burke, E. (2004). Reflections on the Revolution in France. London: Penguin Books.

Cambio 21 (2013). Museo de la Memoria lanzó audioguía en mapudungun, la lengua mapuche. Cambio 21, 27 de abril 2013.

Cañas, E. (1997). Proceso político en Chile, 1973-1990. Santiago de Chile: Andrés Bello.

Casas, F. (2013). Me parece impactante que no existan trabajos de las Yeguas en el Museo de la Memoria. ¡Eso es homofobia! El Mostrador, 11 de septiembre 2013.

Castaño, M. (2013). "Historia magistra vitae" e imitación: La ejemplaridad política de las historias en Maquiavelo, Ingenium, 27, 43-70.

Cicero, M. T. (1862). De Oratore. Leipzig: B.G. Teubner.

Clavel, P. (2007). En 'En 2009, Chile tendrá un Museo de la Memoria en el sector Matucana'. El Mercurio, 24 de mayo de 2007.

de Jouvenel, B. (1959). Introduction to Thucydides. The Peloponnesian War. In Thucydides. The Peloponnesian $W$ ar. The Thomas Hobbes Translation (pp. v-xiv). Ann Arbor: The University of Michigan Press.

Derrida, J. (2006). El siglo y el perdón. Buenos Aires: Ediciones de la Flor.

Durkheim, E. (2003). Les formes élémentaires de la vie religieuse. Paris : PUF.

El Mercurio (2010). Directores de Museo de la Memoria sostienen tenso debate sobre incluir período de Allende y golpe militar. El Mercurio, 6 de enero 2010.

El Mostrador (2010). Manifestación de activistas mapuches empaña inauguración del Museo de la Memoria. El Mostrador, 11 de enero 2010.

Farías, V. (2010). Museo de la Memoria II. El Mercurio, 11 de enero 2010.

Farías, V. (2012a). Museo de la Memoria. El Mercurio, 29 de junio 2012.

Farías, V. (2012b). Museo de la Memoria. El Mercurio, 5 de julio 2012.

Fine, R. (2012). Thoughts on the Legitimacy of Human Rights. In A. Mascareño \& K. Araujo (Eds.), Legitimization in World Society (pp. 25-44). Surrey: Ashgate Publishing.

Garretón, M.A. (2004. Mitos y realidades de la transición democrática en Chile: temas pendientes. Némesis, 4(4): 19-22. 
Habermas, J. (1992). Faktizität und Geltung. Frankfurt aM: Suhrkamp.

Habermas, J. (1995). Die Normalität einer Berliner Republik. Frankfurt aM: Suhrkamp.

Habermas, J. (2001). Die postnationale Konstellation. Frankfurt aM: Suhrkamp.

Habermas, J. (2008). The Constitutionalization of International Law and the Legitimation Problems of a Constitution for World Society. Constellations, 15(4), 444-455.

Halbwachs, M. (1997). La mémoire collective. Paris : Albin Michel.

Hobbes, T. (1981). Leviathan. London: Penguin.

Hobsbawm, E. \& Ranger, T. (Eds.). (1983). The Invention of Tradition. Cambridge: Cambridge University Press.

Hoffman, M. (2008). Lernen aus Katastrophen. Berlin: Sigma.

Kastner, F. (2012). Selbstbeschreibungen ohne Selbst: Gesellschaftliche Umbrüche, Vergangenheitsbewältigung und globaler Prozesse normativer Strukturbildung aus systemtheoretischer Perspektive. In P. Birle, M. Dewey, \& A. Mascareño (Eds.), Durch Lubmanns Brille. Herausforderungen an Politik und Recht in Lateinamerika und in der Weltgesellschaft (pp. 77-98). Wiesbaden: Springer VS.

Kastner, F. (2015). Transnational Justice in der Weltgesellschaft. Hamburg: Hamburger Edition

Koselleck, R. (1992). Vergangene Zukunft. Frankfurt aM: Suhrkamp.

Koselleck, R. (2003). Zeitschichten. Frankfurt aM: Suhrkamp.

Koselleck, R. (2004). Die Herausbildung des modernen Geschichtsbegriffs; Geschichte als moderne Leitbegriff; Ausblick. In R. Koselleck, O. Brunner, \& W. Conze (Eds.), Geschichtliche Grundbegriffe: historisches Lexikon zur politisch-sozialen Sprache in Deutschland. Band 4 (pp. 647-717). Stuttgart: Klett Cotta.

Krebs, M. (2012a). Museo de la Memoria. El Mercurio, 23 de junio 2012.

Krebs, M. (2012b). Museo de la Memoria. El Mercurio, 27 de junio 2012.

Kunreuther, H. \& Useem, M. (2010). Learning from Catastrophes. New Jersey: Prentice Hall.

Lemebel, F. (2013). Pedro Lemebel: 'Mi escritura es estrategia de sobrevivencia más que novelería o letra de vitrina'. El Mostrador, 9 de noviembre 2013.

Luhmann, N. (1996). Zeit und Gedächtnis. Soziale Systeme, 2(2), 307-331.

Luhmann, N. (1997). Die Gesellschaft der Gesellschaft. Frankfurt aM: Suhrkamp.

Mascareño, A. (2012). The Many Faces of Justice. In K. Araujo \& A. Mascareño (Eds.), Legitimization in World Society (pp. 115-136). Surrey: Ashgate Publishing.

Mascareño, A. (2014). La memoria como proyección de futuro. Transtemporalidad y autotrascendencia en la sociedad moderna. In G. Bustamante \& A. Estefane (Eds.), La agonia de la convivencia (pp. 161-170). Santiago de Chile: RIL.

Meier, C. (2010). Das Gebot zu vergessen und die Unabweisbarkeit des Erinnerns: Vom öffentlichen Umgang mit schlimmer Vergangenheit. München: Seidler Verlag.

Monckeberg, C. (2010). Museo de la Memoria. El Mercurio, 26 de enero 2010.

Municipalidad de Melipilla (2015). En el Museo de la Memoria se presentó la 'Cantata Mapudungun' junto a renombrados artistas. Municipalidad de Melipilla, 19 de octubre 2015. Disponible en: http://www.melipilla.cl/2015/10/19/en-el-museo-de-la-memoria-sepresento-la-cantata-mapudungun-junto-a-renombrados-artistas/ [ 25 de diciembre 2016].

Museo de la Memoria (2016). Sobre el Museo. Disponible en: http://ww3.museodelamemoria.cl/sobre-el-museo/\# [25 de diciembre 2016].

Nassehi, A. (2008). Die Zeit der Gesellschaft. Wiesbaden: VS Verlag.

Olick, J. (2007) The Politics of Regret: On Collective Memory and Historical Responsibility. New York: Routledge.

Palet, E. (2012). Museo de la Memoria. El Mercurio, 25 de junio 2012. 
Palma, N. (2007). En 'En 2009, Chile tendrá un Museo de la Memoria en el sector Matucana'. El Mercurio, 24 de mayo de 2007.

Parada, J. (2012). Museo de la Memoria I. El Mercurio, 26 de junio 2012.

Perrow, C. (1984). Normal Accidents. New York: Basic Books.

Perrow, C. (2007). The Disaster After 9/11. In C. Perrow, The Next Catastrophe (pp. 68-129). Princeton: Princeton University Press.

Piñera, S. (2012). En 'Presidente Piñera realiza sorpresiva visita al Museo de la Memoria y los Derechos Humanos'. La Tercera, 20 de abril 2012.

Radio Uchile (2015). Muestra exhibe imágenes inéditas del pueblo mapuche. Diario Uchile, 29 de julio 2015.

Rawls, J. (2000). The Law of Peoples. Cambridge: Cambridge University Press.

Retamal, J. (2010). Museo de la Memoria. El Mercurio, 24 de enero 2010.

Rillón, S. (2010). Museo de la Memoria II. El Mercurio, 20 de junio 2012.

Rojas, G. (2007). En 'En 2009, Chile tendrá un Museo de la Memoria en el sector Matucana'. El Mercurio, 24 de mayo de 2007.

Ruderer, S. (2010). La política del pasado en Chile 1990-2006: ¿Un modelo chileno? Universum, 25(2), 161-177.

Sepúlveda, M.L. (2010). En 'Directores de Museo de la Memoria sostienen tenso debate sobre incluir período de Allende y golpe militar'. El Mercurio, 6 de enero de 2010.

Sepúlveda, M.L.; Rojas, M.E.; Fontaine, A.; Gómez, G.; Ivelic, M.; Montes, F.; Nash, C.; Palet, E.; Peña, C.; Platovsky, D.; Romero, M.; Scantlebury, M.; Squella, A. \& Tohá, C. (2012). Museo de la Memoria. El Mercurio, 28 de junio 2012.

Serrano, S. (2007). En 'La memoria incómoda'. El Mercurio, 16 de junio de 2007.

Simmel, G. (2009). Sociology. Inquiries into the Construction of Social Forms. Boston: Brill.

Squella, A. (2012a). Museo de la Memoria. El Mercurio, 3 de julio 2012.

Squella, A. (2012b). Museo de la Memoria. El Mercurio, 9 de julio 2012.

Topper, B. \& Lagadec, P. (2013). Fractal Crises - A New Path for Crisis Theory and Management. Journal of Contingencies and Crisis Management, 21(1), 4-16.

Ugarte, J. (2010). Museo de la Memoria. El Mercurio, 30 de enero 2010.

Viera-Gallo, J. (2012). Memoria e historia. El Mercurio, 28 de junio 2012.

Villalobos, S. (2012a). Museo de la Memoria. El Mercurio, 22 de junio 2012.

Villalobos, S. (2012b). Museo y odio. El Mercurio, 11 de julio 2012.

Villalobos, S. (2014). Sergio Villalobos vuelve a hacer pebre al Museo de la Memoria y lo acusa de 'culto totémico no razonado'. The Clinic, 19 de enero 2014.

\section{SOBRE EL AUTOR}

Mauro Basaure. PhD en Filosofía, Universidad Johann Wolfgang Goethe (Alemania). Profesor de la Escuela de Sociología y Director del Doctorado en Teoría Crítica y Sociedad Actual de la Universidad Andrés Bello, Chile. Investigador Asociado de la línea Conflicto Político y Social y Coordinador de la Agenda Teórica de COES, Chile.

CONTACTO

mauro.basaure@gmail.com 
142 | Mauro Basaure

Recibido: mayo 2017

Aceptado: agosto 2017

REVISTA MAD | MAGÍSTER EN ANÁLISIS SISTÉMICO APLICADO A LA SOCIEDAD

ISSN 0718-0527

Departamento de Antropología

Facultad de Ciencias Sociales

Universidad de Chile

E. Avenida Capitán Ignacio Carrera Pinto 1045 Nuñoa 7800284 | Santiago | Chile

(o) +56229787760

(a) revistamad.uchile@facso.cl

(\$) www.revistamad.uchile.cl

$\rightarrow$ RevMadUChile Twitter | Facebook 